\title{
REVIEW
}

\section{Alveolar soft-part sarcoma: a review and update}

\author{
A L Folpe, A T Deyrup
}

J Clin Pathol 2006;59:1127-1132. doi: 10.1136/icp.2005.031120

Alveolar soft-part sarcoma (ASPS) is a rare, distinctive sarcoma, typically occurring in young patients. Although it displays a relatively indolent clinical course, the ultimate prognosis is poor and is often characterised by late metastases. Recently, our understanding of the genetic events underlying the pathogenesis of ASPS has greatly increased. The historical, histopathological, ultrastructural, immunohistochemical and genetic aspects of ASPS are reviewed in this article.

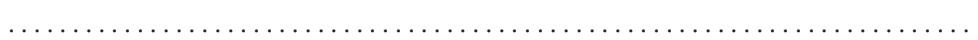

See end of article for authors' affiliations

......................

Correspondence to: A L Folpe, Department of Laboratory Medicine and Pathology, Mayo Clinic, Rochester, Minnesota, USA; folpe.andrew@ mayo.edu

Accepted for publication 25 January 2006
A lveolar soft-part sarcoma (ASPS) is a rare, distinctive sarcoma, typically occurring in young patients. Despite a relatively indolent clinical course, the prognosis is poor and is often characterised by late metastases. Since its formal description by Christopherson et al at Memorial Sloan Kettering Cancer Center, New York, USA, ${ }^{1}$ ASPS has been the subject of considerable interest for pathologists and clinicians, owing to its unique microscopic features, uncertain line of differentiation and unpredictable clinical behaviour. In the past several years, our understanding of the genetic events underlying the pathogenesis of ASPS has greatly increased. This article reviews the historical, histopathological, ultrastructural, immunohistochemical and genetic aspects of ASPS.

\section{HISTORICAL ASPECTS}

Credit for the original description of ASPS traditionally goes to Christopherson, then a fellow in surgical pathology, working under the direction of Drs Foote and Stewart. ${ }^{1}$ In his seminal series of 12 cases taken from the archives of Memorial Sloan Kettering Cancer Center over a 17-year period, Christopherson et al coined the descriptive term "alveolar soft-part sarcoma" for a distinctive and hitherto unrecognised soft-tissue tumour. This tumour typically occurred in the extremities of young women and followed a prolonged clinical course with frequent late metastases. It was defined histologically by the presence of an organoid to pseudoalveolar pattern and large, eosinophilic tumour cells. Interestingly, Christopherson et al noted that several examples of ASPS seemed to have been previously published under a variety of other names, including "malignant myoblastoma", "granular cell myoblastoma" and "malignant granular cell myoblastoma". ${ }^{2-6}$ Although Christopherson et al did not describe the intracytoplasmic crystalline structures that have become one of the hallmarks of ASPS, they did quote an unpublished letter from Pierre Masson at the very end of their discussion, who noted that "trichrome stains show erythrophilic and cyanophilic inclusions. The latter have in places a crystalline structure." ${ }^{11}$ Credit for the seminal description of these crystals thus belongs to Dr Masson, who also showed a periodic acid-Schiff stain of those crystals in his 1956 text, Tumeurs humaines: histologie, diagnostics et techniques. ${ }^{7}$

Apparently unknown to Christopherson and colleagues, ASPS had been described one year previously by Smetana and Scott in a series of 14 cases retrieved from the archives of the Armed Forces Institute of Pathology, as malignant tumours of non-chromaffin paraganglia. A careful reading of this study leaves little doubt that the cases reported by these authors were uniformly histologically and clinically identical to those later reported by Christopherson et al. Smetana and Scott chose the term malignant tumours of nonchromaffin paraganglia because the tumours resembled non-physiologically active paraganglia, postulating that primitive paraganglia-like structures may perhaps normally occur in the somatic soft tissues (a hypothesis since discredited). Smetana and Scott also independently noted the crystals of ASPS, writing that "within the cytoplasm of some of the neoplastic cells were rodshaped, coarse, basophilic bodies of unknown nature ...". ${ }^{8}$

\section{HISTOPATHOLOGICAL FEATURES}

ASPS varies little in its appearance from case to case, and is in some respects remarkable among soft-tissue neoplasms for the absence of described variants. As originally described by Christopherson et $a l^{1}$ (and by Smetana and $\left.\mathrm{Scott}^{8}\right)$, ASPS is characterised by uniform, organoid nests of polygonal tumour cells, separated by fibrovascular septa and delicate capillary-sized vascular channels (fig 1). In these nests there is prominent cellular dyscohesion, leading to the distinctive pseudoalveolar pattern for which it is named. The organoid appearance may be completely lost and the tumour may be composed of sheets of epithelioid cells. Tumours occurring in younger patients and in confined locations, such as the tongue, often show very small nests of cells, closely mimicking true paragangliomas 9 (fig 2). Intravascular tumour extension is seen in most cases (fig 3). A small minority of tumours shows unusual features such as myxoid change, cystic change, haemorrhage and a prominent lymphocytic infiltrate ${ }^{18-20}$ (fig 4).

The cells in the lesion have distinct borders and abundant eosinophilic to clear, somewhat

Abbreviations: ASPS, alveolar soff-part sarcoma; TFE3, transcription factor 3 


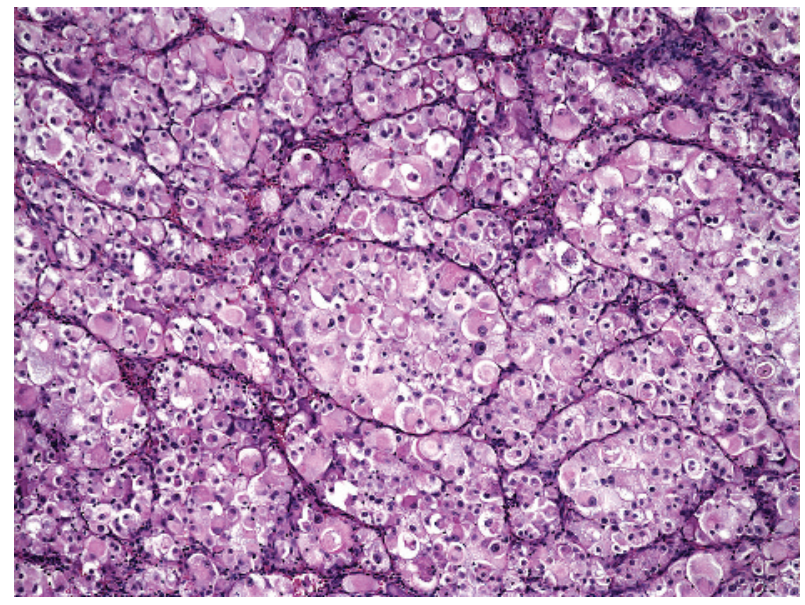

Figure 1 Low-power view of a typical alveolar soff-part sarcoma, showing an organoid, pseudoalveolar proliferation of large, eosinophilic cells and a delicate capillary network.

granular, cytoplasm, resulting in an epithelioid appearance (fig 5). Clear cell change may be very prominent, closely mimicking renal cell carcinoma ${ }^{21}$ (fig 6). Cross striations and cytoplasmic fibrils are absent. A characteristic finding is the presence of periodic acid-Schiff-positive, diastase-resistant crystalline structures, which may be rhomboid, rod-like or spiked, in individual, sheaf-like or stacked configurations. ${ }^{10}$ These crystals can also be identified with alcian blue and trichrome histochemical stains. These crystals are not especially prominent in all cases, and multiple sections may require careful searching to identify them. ASPS cells typically have round, regular, eccentrically placed nuclei with vesicular chromatin and a prominent central nucleolus; multinucleation may be present in a few cells (fig 7). Mitotic activity is usually low and necrosis is infrequent.

Although this description applies to most ASPSs, rare cases may deviate from a typical ASPS by virtue of the presence of anaplastic-appearing tumour cells with marked variation in nuclear size. ${ }^{1520}$ As noted by Evans, ${ }^{20}$ these anaplasticappearing foci may also show other atypical features, such as sheet-like growth, frequent mitotic figures and coagulative tumour cell necrosis. Extremely rare cases with spindling and xanthomatous change have also been described. ${ }^{15} 22$

\section{Ultrastructural features}

The first ultrastructural study on ASPS was that by Shipkey et $\mathrm{al}^{10}$ in 1964, with subsequent important studies over the next 25 years by Font et al, ${ }^{23}$ DeSchryver-Kecskemeti et al, ${ }^{24}$ Mukai et $a l^{25}$ and Ordonez et $a l^{12}$ among others. ASPS cells typically rest on an incomplete basement membrane and contain only rare, primitive cell-cell junctions. The cytoplasm contains numerous mitochondria and an extensive Golgi complex with adjacent small dense granules. ${ }^{10}{ }^{12} 23-25$ The endoplasmic reticulum is usually, but not always, sparse. The distinctive crystals of ASPS are typically found intermingled with these dense granules. They are often bounded by a single membrane and are composed of a periodic latticework of fibres (diameter $4.5-5 \mathrm{~nm}$ and periodicity $10 \mathrm{~nm}$; fig 8). ${ }^{10}{ }^{12} 23-25$ The composition of these crystals was interpreted as a product similar to renin ${ }^{24}$ or aggregates of actin filaments, ${ }^{25}$ and it was suggested that they could be similar to the inclusions seen in rhabdomyoma or nemaline rod myopathy. ${ }^{26}$ However, elegant recent work by Ladanyi et $\mathrm{al}_{,}{ }^{27}$ including ultrastructural immunohistochemistry, has convincingly shown that the crystals consist of aggregates of the monocarboxylate transporter protein MCTl and its cellular chaperone CD147. ${ }^{27}$

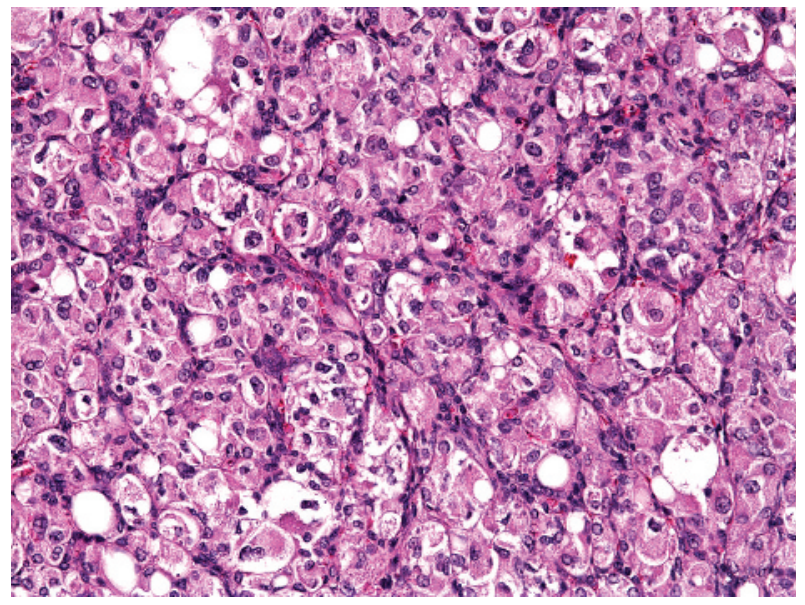

Figure 2 Low-power view of an alveolar soft-part sarcoma with a uniform "small nest" pattern. Such cases may easily be mistaken for paragangliomas.

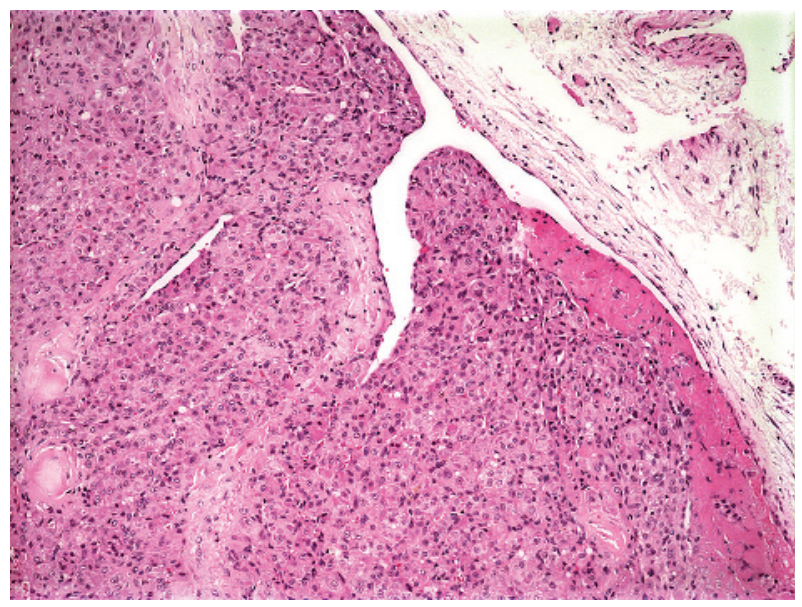

Figure 3 Intravascular extension in an alveolar soft-part sarcoma (ASPS); this is present at the periphery of the tumour in nearly all cases when examined closely, and may account for the high rate of metastasis seen in the ASPS.

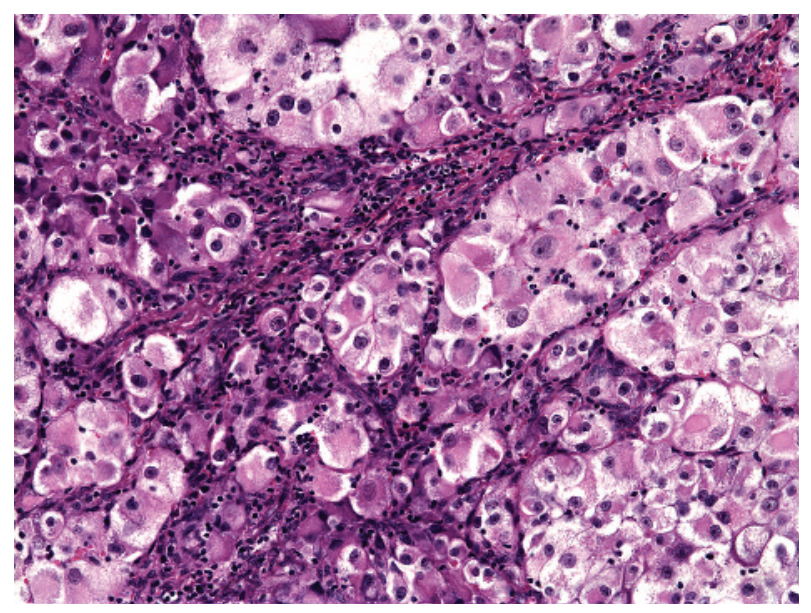

Figure 4 Alveolar soft-part sarcoma with a prominent associated chronic inflammatory cell infiltrate. 


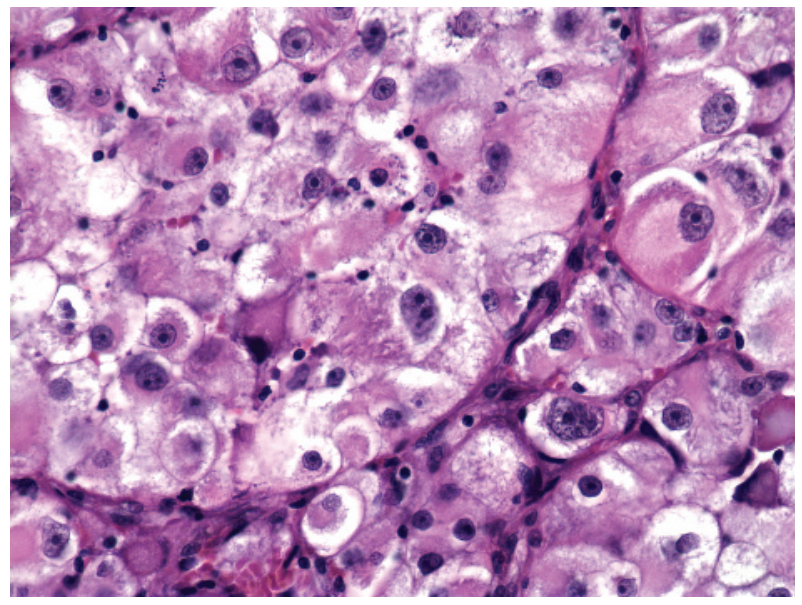

Figure 5 High-power view of a typical alveolar soft-part sarcoma, showing relative cellular uniformity and typical cytological features, with peripherally placed, vesicular nuclei containing a prominent central nucleolus.

\section{IMMUNOHISTOCHEMICAL FINDINGS}

Until recently, immunohistochemical analysis played a limited part in the diagnosis of ASPS. Several studies have examined ASPS immunohistochemically, both with and without the use of epitope-retrieval techniques, often with differing results (reviewed by Foschini and Eusebi ${ }^{28}$ ). In general, ASPSs are negative for epithelial markers, such as cytokeratins and epithelial membrane antigen, negative for specific neuroendocrine markers such as chromogranin A and synaptophysin, and negative for specific melanocytic markers, such as HMB45 and Melan-A. Non-specific markers such as neurone-specific enolase and vimentin may be present in roughly $30-50 \%$ of cases. There has been a great deal of interest in the expression of muscle-related proteins in ASPS, due to the prevailing theory for many years that ASPS represented an unusual form of myogenic tumour. Antibodies to actins in pan, smooth and skeletal muscle have been reported to be positive in nearly $50 \%$ of patients with ASPS. Actin expression in pan and smooth muscle is by no means specific for myogenous differentiation, and the ASR-1 antibody to skeletal-muscle actins is notoriously difficult to interpret because of high levels of non-specific staining. Desmin expression has been reported in around 50\% of cases with ASPS, although it tends to be present in only a small number of the neoplastic cells. Again, it should be emphasised that desmin expression is not limited to myogenous tumours and can be seen in a wide variety of other lesions, including melanoma, tenosynovial giant-cell tumour, Ewing's sarcoma and angiomatoid "malignant" fibrous histiocytoma, among others. ${ }^{21}$

A much more controversial topic has been whether ASPS expresses truly specific markers of skeletal muscle differentiation, such as the myogenic nuclear regulatory proteins MyoDl and myogenin. In 1991, Rosai et al $^{29}$ reported a case of ASPS that seemed to show convincing MyoDl expression by immunofluorescence and western blotting on fresh-frozen tissue. ${ }^{29}$ This was followed by Tallini et al's $\mathrm{s}^{30}$ report on a MyoDl-positive ASPS, using immunohistochemistry on frozen sections. Regrettably, these two early findings have not been substantiated by any subsequent study. Three separate studies, with commercially available antibodies and modern immunohistochemical techniques, including heat-induced epitope retrieval, have not identified expression of either MyoDl or myogenin in the more than 35 cases studied. ${ }^{31-33}$

It has recently been discovered that ASPSs are characterised by a tumour-specific $\operatorname{der}(17) \mathrm{t}(\mathrm{X} ; 17)(\mathrm{p} 1 \mathrm{l} ; \mathrm{q} 25)$ that fuses the

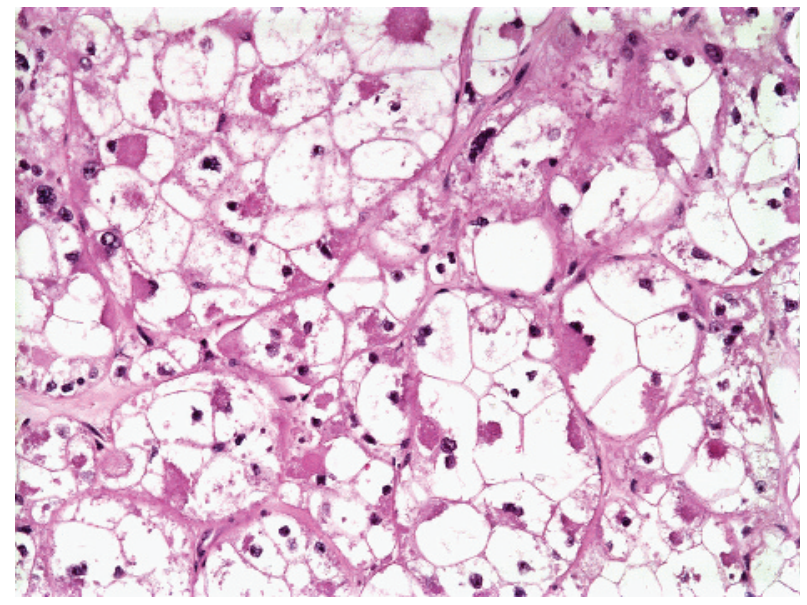

Figure 6 High-power view of an alveolar soft-part sarcoma, with uniform clear cell change. This is believed to be an artefact related to poor cell preservation. However, it may closely simulate metastatic renal cell carcinoma of conventional subtype.

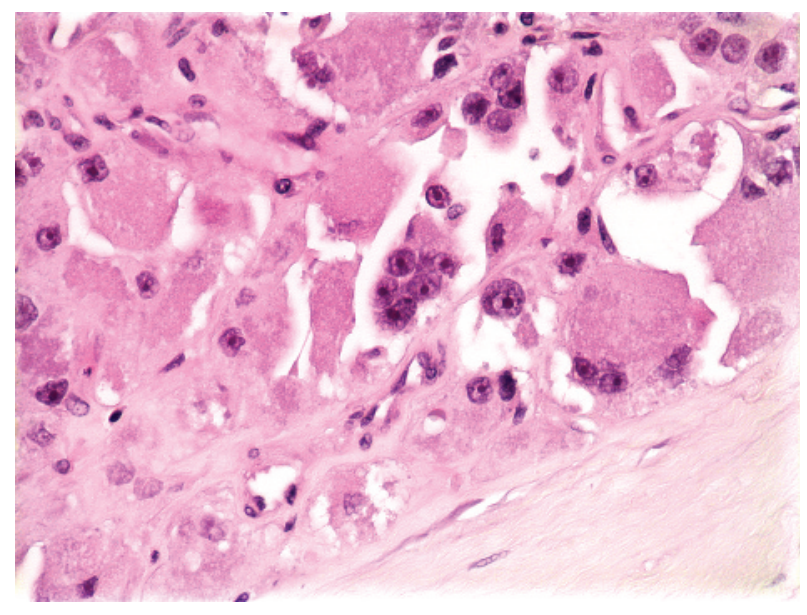

Figure 7 Multinucleation in an alveolar soft-part sarcoma, a relatively frequent finding.

transcription factor 3 (TFE3) gene at Xpll to the ASPL gene at 17q25, creating an ASPL-TFE3 fusion protein. ${ }^{34}$ Recently, an antibody directed against the C-terminus of the TFE3 has emerged as a highly sensitive and specific marker of the ASPS ${ }^{35}$ (fig 9). Although TFE3 seems to be almost universally expressed in normal tissues, this expression is at very low levels and strong nuclear expression of TFE3 is seen almost exclusively in tumours known to contain the TFE3 gene fusions, such as ASPSs and rare paediatric renal carcinomas. ${ }^{35}$ It must be emphasised that only nuclear expression of TFE3 is of diagnostic value, as cytoplasmic staining (possibly non-specific) is seen in various tumours. The TFE3 antibody binds to the protein downstream of exon 4 and therefore is positive in both fusion variants seen in ASPS. Importantly, however, strong TFE3 is seen in granular cell tumours, ${ }^{35}$ a potentially confusing and somewhat ironic finding, given the original classification of ASPS as a "malignant granular cell myoblastoma" (malignant granular cell tumour).

\section{CYTOGENETIC AND MOLECULAR GENETIC FEATURES}

The earliest cytogenetic analysis of ASPS identified multiple structural and numerical abnormalities, strongly suggesting a role for chromosomal abnormalities in the pathogenesis of 


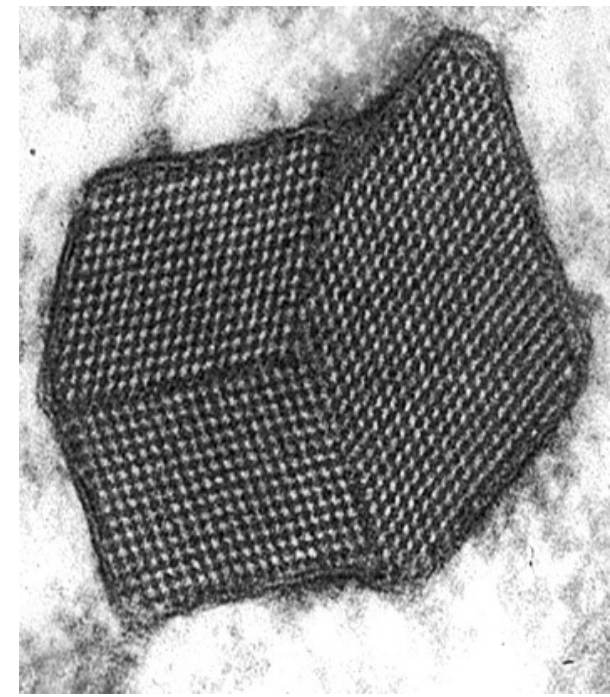

Figure 8 Electron micrograph, showing a typical intracyłoplasmic crystal in an alveolar soft-part sarcoma (reproduced with the permission of Dr Cyril Fisher, The Royal Marsden NHS Trust, London, UK).

this tumour. ${ }^{36}$ Subsequent karyotypes identified translocations between chromosomes X and $17^{37-41}$ and, in 1998, both break points were identified: $17 \mathrm{q} 25$ and Xp11.2. ${ }^{42}$ Subsequently, Ladanyi et $a l^{34}$ characterised the two genes concerned in the translocation.

ASPS is characterised by an unbalanced translocation: $\operatorname{der}(17) t(X: 17)(p 11 ; p 25)$. This translocation results in the fusion of a gene of unknown function, ASPL, on chromosome 17 to the TFE3 gene on the $\mathrm{X}$ chromosome. ${ }^{34}$ Consequently, the ASPL gene is joined in frame upstream of either the third or fourth exon of TFE3, yielding two fusion variants, type 1 and type 2, respectively (fig 10). Usually, fusion genes are generated by reciprocal balanced translocations with only one of the two chimeric genes being pathogenic. The translocation in ASPS is unusual in that it is unbalanced, although two patients with a reciprocal translocation have been described. $^{34}{ }^{43}$ It has been suggested that the female predominance seen in patients with ASPS is because of the presence of two $\mathrm{X}$ chromosomes in these patients, increasing their chances of a translocation on this chromosome. ${ }^{34}$

ASPL is a ubiquitously expressed protein with greatest expression in adult heart, skeletal muscle, pancreas and testis, and somewhat lower expression in fetal tissues. ${ }^{34}$ Although little is known about the ASPL protein, the gene itself has been partially characterised: the cDNA is 1872 nucleotides in length, yielding a predicted protein of 476 amino acids. Sequence comparison with known human genes has not identified major homology; however, there is some similarity to sequences from other species, including yeast, fruit fly and worm; ${ }^{34}$ the protein function is unknown in all of those species. At the structural level, a sequence homologous to the UBX motif was found in the C-terminus of ASPL. ${ }^{34}$ The UBX domain is an 80-amino acid module localised in the C-terminus of several eukaryotic proteins and is hypothesised to have a role in ubiquitin-related processes ${ }^{44}$ No other complex domains have been identified in the ASPL sequence.

TFE3 is much more understood: it is one of a family of basic helix-loop-helix leucine zipper transcription factors that includes MiTF, TFEB and TFEC..$^{45}$ They are ubiquitously expressed and bind DNA as homodimers and heterodimers. All possible pairwise combinations have been identified in vitro, and it has been suggested that tissue-specific gene expression is regulated by the relative coexpression of each protein. ${ }^{45}$ Two activation domains have been identified in TFE3:

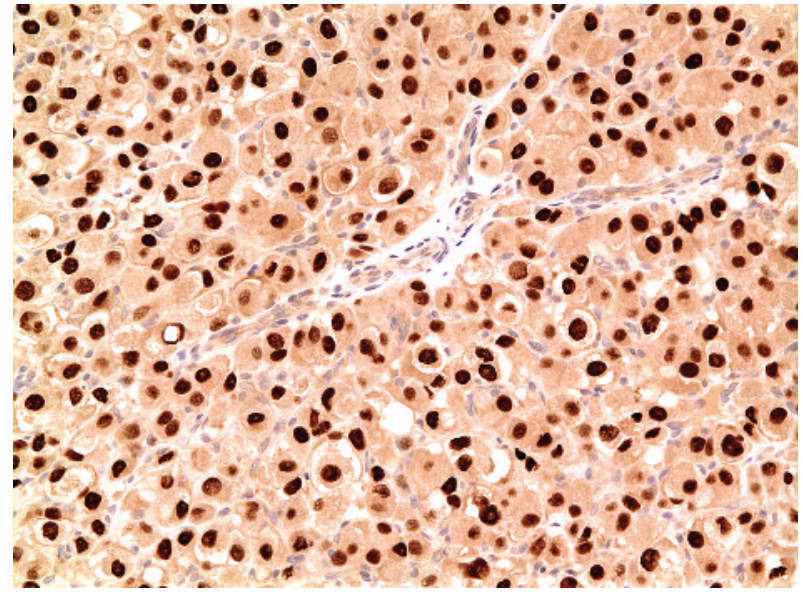

Figure 9 An alveolar soft-part sarcoma (ASPS) showing uniform, strong nuclear positivity with an antibody directed against the $\mathrm{C}$-terminus of transcription factor 3 (TFE3), confirming the presence of an ASPLTFE3 fusion protein.

TFE3
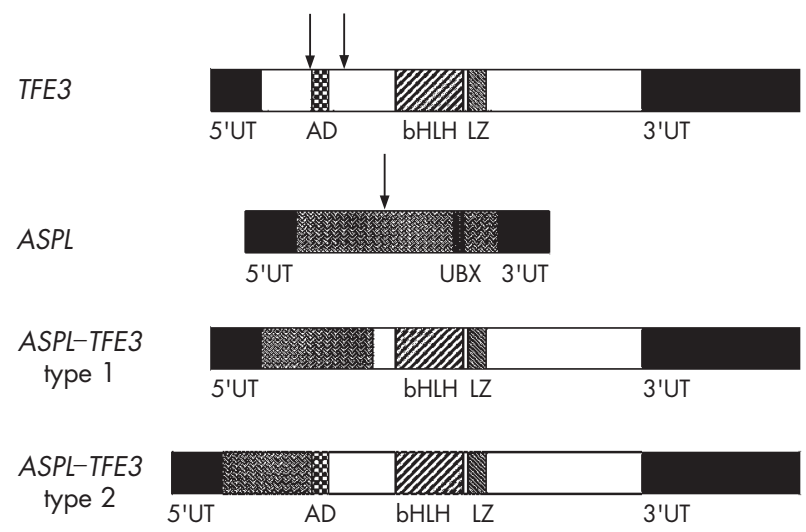

Figure 10 Structure of the alveolar soft-part sarcoma (ASPL), locus gene transcription factor 3 (TFE3) gene and the ASPL-TFE3 fusion gene. $A D$, activation domain; bHLH, basic helix-loop-helix; LZ, leucine zipper; UT, uridine triphosphate.

an acidic, N-terminal domain and a proline-rich C-terminal domain. ${ }^{46}$ These two domains act synergistically and differential splicing provides additional regulatory control. The loss of the $\mathrm{N}$-terminal-activating domain, as is seen in fusion variant $\mathrm{l}$, results in a dominant negative form of the transcriptional activator that interferes with full-length TFE3. ${ }^{47}$

The composite ASPL-TFE3 protein loses the ASPL UBX domain but retains the TFE3 basic helix-loop-helix DNAbinding domain, leucine zipper dimerisation domain and Cterminal activation domain. The ubiquitous expression of ASPL suggests constitutive expression of the ASPL promoter and, accordingly, it has been hypothesised that the loss of the TFE3 upstream regulatory elements may contribute to the pathogenesis of this translocation..$^{34}$ In the type 2 fusion, the $\mathrm{N}$-terminal TFE3-activating domain is lost and, although no clinical difference has been identified between the two fusion variants, only a few cases have been analysed. ${ }^{34} 43$

Interestingly, TFE3 is partnered with several other genes in balanced reciprocal translocations in a subset of (usually) paediatric papillary renal cell adenocarcinomas. ${ }^{48-52}$ In these tumours, several possible translocations can be seen: (1) the entire coding region of TFE 3 is fused with the novel gene PRCC at lq21.2 or (2) TFE3 minus the first three exons is fused to one of two splicing factor genes, PSF at $1 \mathrm{p} 34$ or NonO 
at Xq12. The second case is analogous to that seen in ASPLTFE3 type 1 fusion as, in both instances, the TFE3 activation domain is lost, whereas the basic helix-loop-helix DNAbinding domain is retained.

\section{CLINICAL FEATURES AND BEHAVIOUR}

ASPS is extremely rare, and is generally believed to account for $<1 \%$ of soft-tissue tumours overall. ${ }^{21}$ ASPS has, however, accounted for roughly $10 \%$ of cases reported from large sarcoma referral centres, in both adults ${ }^{18}$ and children. ${ }^{14}$ As many as $60 \%$ of ASPS cases are seen in women, ${ }^{18}$ although this female sex predilection may be less pronounced in children. ${ }^{14} 17$ The tumour typically occurs in the deep soft tissues, most often in the buttock and thigh, with a smaller number of cases at other soft-tissue locations such as the arm, chest and retroperitoneal tissues. ${ }^{18-20}$ In children, a substantial percentage of cases occur in the head and neck, often in the orbit ${ }^{23}$ or tongue. ${ }^{9}$ ASPSs have been reported in a wide variety of locations, including the bladder, ${ }^{6}$ stomach, ${ }^{53}$ gynaecological tract ${ }^{54}$ and bone. ${ }^{55}$ Most often, ASPSs present as painless masses, which may be highly vascular on imaging studies. ${ }^{21}$

ASPS behaves as a relatively indolent, but relentless sarcoma, characterised by late metastases and an extended clinical course; survival rates of $77 \%$ at 2 years, $60 \%$ at 5 years, $38 \%$ at 10 years and only $15 \%$ at 20 years have been reported by Lieberman et al. ${ }^{18}$ Studies on another large series from the MD Anderson Cancer Center, Texas, USA, found a 5 -year disease-free survival of $71 \%$ in patients presenting with localised disease as compared with only $20 \%$ in patients presenting with metastases. ${ }^{56}$ The prognosis for children with ASPS may be considerably better, with Pappo et $\mathrm{al}^{14}$ reporting disease-free survival in 9 of 11 cases (with a relatively short follow-up duration) and Casanova et al ${ }^{17}$ reporting $100 \%$ survival at $>5$-year follow-up in 12 patients with localised disease at presentation. Lingual and orbital tumours also have very high survival rates, possibly reflecting a combination of small size at the time of diagnosis and younger patient age. ${ }^{923}$

No histopathological features are predictive of prognosis in patients with ASPS, and these tumours should not be graded under either the National Cancer Institute or French grading schemes. $\mathrm{NCI}^{57}$ Features associated with improved prognosis include younger age at diagnosis, small tumour size and the presence of localised disease. ${ }^{1856}$ ASPSs most often metastasise to lungs, brain and bone. Metastases, sometimes to unusual locations such as the breast, may be the presenting symptom in some patients with ASPS. Adjuvant chemotherapy does not seem to be effective in the treatment of ASPSs, although there may be some role for adjuvant radiotherapy in reducing the risk for local recurrences. ${ }^{18} 5658$

\section{LINE OF DIFFERENTIATION}

The cell of origin or, better, line of differentiation taken by ASPS has been the subject of considerable speculation and controversy over the past 50 years. ASPS was originally regarded as a malignant variant of granular cell myoblastoma, a term that encompasses the entity now known as granular cell tumour. ${ }^{2-6575960}$ The speculation that ASPSs represented unusual malignant tumours of non-chromaffin paraganglia $^{8}$ has subsequently been disproved by several ultrastructural, histochemical and immunohistochemical studies, documenting the complete absence of neuroendocrine differentiation in ASPS. ${ }^{25}{ }^{61}$ Similarly, the suggestion that ASPSs were malignant angioreninomas has been disproved by negative immunohistochemical studies for renin, as well as by the absence of clinical signs of hyperreninism in patients with ASPS. ${ }^{25}$ Perhaps the longest lived hypothesis has been that ASPS is an unusual myogenous tumour, on the basis of some ultrastructural similarity of a component of the ASPS crystal to actin filaments, ${ }^{25}$ overlap between the appearance of these crystals and those seen in nemaline myopathy and rhabdomyoma, ${ }^{26}$ and variable expression of muscle-related proteins, such as actins and desmin. $^{28}$ However, as discussed in the section Immunohistochemical findings, the specificity and significance of these immunohistochemical findings have been questioned, and there is no convincing evidence of expression of truly muscle-specific proteins in ASPS. ${ }^{62}$

In the light of the recent discovery that ASPS is part of the family of translocation-associated sarcomas, there exists an additional hypothesis-that ASPS displays a scrambled phenotype, without a normal counterpart. ${ }^{63}$ This would seem to be a compelling hypothesis, given the lack of resemblance of ASPS to any known structure, its resolute defiance of all attempts to categorise it and our increasing understanding that a subset of human neoplasms, such as perivascular epithelioid cell neoplasms, possibly lack normal counterparts. ${ }^{57}$ Alternatively, it cannot be entirely excluded that ASPS recapitulates the phenotype of a yet-to-be discovered, extremely rare, normal cell of unknown function.

\section{DIFFERENTIAL DIAGNOSIS}

The differential diagnosis of ASPS is fairly broad, and revolves around neoplasms that may show nested or organoid patterns of growth and cells with abundant eosinophilic cytoplasm. Renal cell carcinomas, adrenal cortical carcinomas and hepatocellular carcinomas may mimic ASPS by virtue of their abundant eosinophilic to clear cytoplasm. Immunohistochemical evidence of strong cytokeratin expression, expression of site-associated markers (eg, renal cell carcinoma antigen in renal cell carcinoma, Melan-A crossreactivity in adrenal cortical carcinoma and HepParl in hepatocellular carcinoma) and absence of TFE3 expression should help in this differential diagnosis. Paediatric renal cell carcinomas with TFE3 gene fusions may show cytokeratin expression and TFE3 expression to be absent; clinical correlation as to the presence of a renal mass is critical in this situation. Malignant melanoma may simulate ASPS, but should be easily separated immunohistochemically for S100 protein, HMB45 and Melan A. Paragangliomas, unlike ASPSs, show strong expression of chromogranin A and synaptophysin. Alveolar rhabdomyosarcoma, despite its somewhat similar name, appears as an entirely different "small blue round cell tumour", which strongly expresses desmin and myogenin nuclear regulatory proteins. Perivascular epithelioid cell neoplasms, such as epithelioid angiomyolipoma, coexpress smooth-muscle actin and melanocytic markers, and are almost always TFE3-negative. In most instances, granular cell tumours lack the striking cytological atypia seen in ASPS and show strong S100 protein expression. TFE3, however, may be expressed by granular cell tumours, a potential pitfall.

\section{CONCLUSIONS}

ASPSs are rare soft-tissue sarcomas with a distinctive and generally invariable histological appearance. It is now clear that they are caused by a specific unbalanced translocation, $\operatorname{der}(17) \mathrm{t}(\mathrm{X}: 17)(\mathrm{pl} 1 ; \mathrm{p} 25)$, which results in the formation of an ASPL-TFE3 fusion gene. The presence of this fusion gene can be detected by either molecular genetics or immunohistochemical analysis for the C-terminus of the ASPL-TFE3 fusion protein, assisting in the diagnosis of ASPS and in its distinction from the wide variety of other neoplasms with which it may be confused. The presence of this unique fusion gene also suggests that ASPS lacks a normal cellular counterpart. 


\section{Authors' affiliations}

A L Folpe, Department of Laboratory Medicine and Pathology, Mayo Clinic Rochester, Minnesota, USA

A T Deyrup, Department of Pathology and Laboratory Medicine, Emory University Hospital, Atlanta, Georgia, USA

Competing interests: None declared.

\section{REFERENCES}

1 Christopherson WM, Foote FW Jr, Stewart FW. Alveolar soft-part sarcomas; structurally characteristic tumors of uncertain histogenesis. Cancer 1952;5:100-11.

2 Ackerman LV, Phelps CR. Malignant granular cell myoblastoma of the gluteal region. Surgery 1946;20:511-9.

3 Horn RC, Stout AP. Granular cell myoblastoma. Surg Gynecol Obstet 1943:76:315-8.

4 Khanolkar VR. Granular cell myoblastoma. Am J Pathol 1947;23:721-39.

5 Klemperer P. Myoblastoma of the striated muscle. Am J Cancer 1934:20:324-37.

6 Ravich A, Stout AP, Ravich RA. Malignant granular cell myoblastoma involving the urinary bladder. Ann Surg 1945;121:361-72.

7 Masson P. Tumeurs humaines: histologie, diagnostics et techniques, 2nd edn. Paris: Libraire Maloine, 1956.

8 Smetana HF, Scott WF Jr. Malignant tumors of nonchromaffin paraganglia. Mil Surg 1951;109:330-49.

9 Fanburg-Smith JC, Miettinen M, Folpe AL, et al. Lingual alveolar soft part sarcoma; 14 cases: novel clinical and morphological observations. Histopathology 2004:45:526-37.

10 Shipkey FH, Lieberman PH, Foote FW Jr, et al. Ultrastructure of alveolar soft part sarcoma. Cancer 1964;17:821-30.

11 Auerbach HE, Brooks JJ. Alveolar soft part sarcoma. A clinicopathologic and immunohistochemical study. Cancer 1987;60:66-73.

12 Ordonez NG, Ro JY, Mackay B. Alveolar soft part sarcoma. An ultrastructural and immunocytochemical investigation of its histogenesis. Cancer 1989;63:1721-36

13 Ordonez NG, Hickey RC, Brooks TE. Alveolar soft part sarcoma. A cytologic and immunohistochemical study. Cancer 1988;61:525-31.

14 Pappo AS, Parham DM, Cain A, et al. Alveolar soft part sarcoma in children and adolescents: clinical features and outcome of 11 patients. Med Pediatr Oncol 1996;26:81-4

15 Jong R, Kandel R, Fornasier $V$, et al. Alveolar soft part sarcoma: review of nine cases including two cases with unusual histology. Histopathology 1998;32:63-8

16 Ordonez NG, Mackay B. Alveolar soft-part sarcoma: a review of the pathology and histogenesis. Ultrastruct Pathol 1998;22:275-92.

17 Casanova M, Ferrari A, Bisogno G, et al. Alveolar soft part sarcoma in children and adolescents: a report from the Soft-Tissue Sarcoma Italian Cooperative Group. Ann Oncol 2000;1 1:1445-9.

18 Lieberman PH, Brennan MF, Kimmel M, et al. Alveolar soft-part sarcoma. A clinico-pathologic study of half a century. Cancer 1989:63:1-13.

19 Lieberman PH, Foote FW Jr, Stewart FW, et al. Alveolar soft-part sarcoma. JAMA 1966;198:1047-51.

20 Evans HL. Alveolar soft-part sarcoma. A study of 13 typical examples and one with a histologically atypical component. Cancer 1985;55:912-7.

21 Enzinger FM, Weiss SW. Soft tissue tumors, 3rd edn. St Louis: Mosby, 1995.

22 Wu J, Brinker DA, Haas M, et al. Primary alveolar soft part sarcoma (ASPS) of the breast: report of a deceptive case with xanthomatous features confirmed by TFE3 immunohistochemistry and electron microscopy. Int J Surg Pathol 2005; 13:81-5

23 Font RL, Jurco S 3rd, Zimmerman LE. Iveolar soft-part sarcoma of the orbit: a clinicopathologic analysis of seventeen cases and a review of the literature. Hum Pathol 1982;13:569-79.

24 DeSchryver-Kecskemeti K, Kraus FT, Engleman W, et al. Alveolar soft-part sarcoma-a malignant angioreninoma: histochemical, immunocytochemical, and electron-microscopic study of four cases. Am J Surg Pathol 1982;6:5-18.

25 Mukai M, Iri H, Nakajima T, et al. Alveolar soft-part sarcoma. A review on its histogenesis and further studies based on electron microscopy, immunohistochemistry, and biochemistry. Am J Surg Pathol 1983;7:679-89.

26 Fisher ER, Reidbord H. Electron microscopic evidence suggesting the myogenous derivation of the so-called alveolar soft part sarcoma. Cancer 1971;27:150-9.

27 Ladanyi M, Antonescu CR, Drobnjak M, et al. The precrystalline cytoplasmic granules of alveolar soft part sarcoma contain monocarboxylate transporter 1 and CD147. Am J Pathol 2002;160:1215-21.

28 Foschini MP, Eusebi V. Alveolar soft-part sarcoma: a new type of rhabdomyosarcoma? Semin Diagn Pathol 1994;1 1:58-68.

29 Rosai J, Dias P, Parham DM, et al. MyoD1 protein expression in alveolar soff part sarcoma as confirmatory evidence of its skeletal muscle nature. Am J Surg Pathol 1991;15:974-81.

30 Tallini G, Parham DM, Dias $\mathrm{P}$, et al. Myogenic regulatory protein expression in adult soft tissue sarcomas. A sensitive and specific marker of skeletal muscle differentiation. Am J Pathol 1994; 144:693-701.

31 Wang NP, Bacchi CE, Jiang JJ, et al. Does alveolar soft-part sarcoma exhibit skeletal muscle differentiation? An immunocytochemical and biochemical study of myogenic regulatory protein expression. Mod Pathol 1996;9:496-506
32 Gomez JA, Amin MB, Ro JY, et al. Immunohistochemical profile of myogenin and MyoDI does not support skeletal muscle lineage in alveolar soft part sarcoma. Arch Pathol Lab Med 1999;123:503-7.

33 Cessna MH, Zhou H, Perkins SL, et al. Are myogenin and MyoDl expression specific for rhabdomyosarcoma? A study of 150 cases, with emphasis on spindle cell mimics. Am J Surg Pathol $2001 ; 25: 1150-7$.

34 Ladanyi M, Lui MY, Antonescu CR, et al. The $\operatorname{der}(17)+(X ; 17)(p 11 ; q 25)$ of human alveolar soft part sarcoma fuses the TFE3 transcription factor gene to ASPL, a novel gene at 17q25. Oncogene 2001;20:48-57.

35 Argani $P$, Lal $P$, Hutchinson $B$, et al. Aberrant nuclear immunoreactivity for TFE3 in neoplasms with TFE3 gene fusions: a sensitive and specific immunohistochemical assay. Am J Surg Pathol 2003;27:750-61.

36 Sreekantaiah C, Li FP, Weidner N, et al. Multiple and complex abnormalities in a case of alveolar soft-part sarcoma. Cancer Genet Cytogenet 1991;55:167-71.

37 Cullinane C, Thorner PS, Greenberg ML, et al. Molecular genetic, cytogenetic, and immunohistochemical characterization of alveolar soft-part sarcoma. Implications for cell of origin. Cancer 1992;70:2444-50.

38 van Echten J, van den Berg E, van Baarlen J, et al. An important role for chromosome 17, band $q 25$, in the histogenesis of alveolar soft part sarcoma. Cancer Genet Cytogenet 1995;82:57-61.

39 Lillehei KO, Kleinschmidt-DeMasters B, Mitchell DH, et al. Alveolar soft part sarcoma: an unusually long interval between presentation and brain metastasis. Hum Pathol 1993:24:1030-4.

40 Sciot R, Dal Cin P, De Vos R, et al. Alveolar soft-part sarcoma: evidence for its myogenic origin and for the involvement of $17 q 25$. Histopathology 1993;23:439-44

41 Heimann P, Devalck C, Debusscher C, et al. Alveolar soft-part sarcoma: further evidence by FISH for the involvement of chromosome band 17q25. Genes Chromosomes Cancer 1998;23:194-7.

42 Joyama S, Ueda T, Shimizu K, et al. Chromosome rearrangement at 17q25 and $\mathrm{xp}$ 1 1. 2 in alveolar soft-part sarcoma: a case report and review of the literature, Cancer 1999:86:1246-50.

43 Uppal S, Aviv H, Patterson F, et al. Alveolar soft part sarcoma-reciprocal translocation between chromosome 17q25 and Xp1 1. Report of a case with metastases at presentation and review of the literature. Acta Orthop Belg 2003:69:182-7.

44 Buchberger A, Howard MJ, Proctor M, et al. The UBX domain: a widespread ubiquitin-like module. J Mol Biol 2001;307:17-24.

45 Kuiper RP, Schepens M, Thijssen J, et al. Regulation of the MiTF/TFE bHLH-LZ transcription factors through restricted spatial expression and alternative splicing of functional domains. Nucleic Acids Res 2004;32:2315-22.

46 Artandi SE, Merrell K, Avitahl N, et al. TFE3 contains two activation domains, one acidic and the other proline-rich, that synergistically activate transcription. Nucleic Acids Res 1995;23:3865-71.

47 Roman C, Cohn L, Calame K. A dominant negative form of transcription activator mTFE3 created by differential splicing. Science 1991;254:94-7.

48 Argani P, Ladanyi M. Recent advances in pediatric renal neoplasia. Adv Anat Pathol 2003;10:243-60.

49 Argani P, Antonescu CR, Illei PB, et al. Primary renal neoplasms with the ASPL-TFE3 gene fusion of alveolar soft part sarcoma: a distinctive tumor entity previously included among renal cell carcinomas of children and adolescents. Am J Pathol 2001;159:179-92.

50 Argani $\mathbf{P}$, Lae $M$, Hutchinson $B$, et al. Renal carcinomas with the $t(6 ; 11)(p 21 ; q 12)$ : clinicopathologic features and demonstration of the specific alpha-TFEB gene fusion by immunohistochemistry, RT-PCR, and DNA PCR. Am J Surg Pathol 2005;29:230-40.

51 Davis IJ, Hsi BL, Arroyo JD, et al. Cloning of an alpha-TFEB fusion in renal tumors harboring the $t(6 ; 11)(\mathrm{p} 21 ; \mathrm{q} 13)$ chromosome translocation. Proc Nat Acad Sci USA 2003;100:6051-6.

52 Clark J, Lu YJ, Sidhar SK, et al. Fusion of splicing factor genes PSF and NonO (p54nrb) to the TFE3 gene in papillary renal cell carcinoma. Oncogene 1997; 15:2233-9.

53 Yaziji H, Ranaldi R, Verdolini R, et al. Primary alveolar soft part sarcoma of the stomach: a case report and review. Pathol Res Pract 2000;196:519-25.

54 Nielsen GP, Oliva E, Young RH, et al. Alveolar soft-part sarcoma of the female genital tract: a report of nine cases and review of the literature. Int J Gynecol Pathol 1995; 14:283-92.

55 Park YK, Unni KK, Kim YW, et al. Primary alveolar soft part sarcoma of bone. Histopathology 1999;35:41 1-7.

56 Portera CA Jr, Ho V, Patel SR, et al. Alveolar soft part sarcoma: clinical course and patterns of metastasis in 70 patients treated at a single institution. Cancer 2001;91:585-91

57 Fletcher CDM, Unni KK, Mertens F, et al. Pathology and genetics of tumours of soft tissue and bone. Lyon: IARC Press, 2002.

58 Sherman N, Vavilala $M$, Pollock R, et al. Radiation therapy for alveolar softpart sarcoma. Med Pediatr Oncol 1994;22:380-3.

59 Corbetta S. Granular cell myoblastoma. Description of a case with malignant evolution and final aspect of alveolar soft part sarcoma. Arch Ital Chir 1967:93:291-301.

60 Fierro FJ, Dorfman HD. Granular cell myoblastoma of the hand: report of three new cases with aggressive behavior in one. Bull Hosp Jt Dis 1975;36:121-9

61 Ekfors TO, Kalimo H, Rantakokko V, et al. Alveolar soft part sarcoma: a repor of two cases with some histochemical and ultrastructural observations. Cancer 1979;43:1672-7.

62 Folpe AL. MyoD1 and myogenin expression in human neoplasia: a review and update. Adv Anat Pathol 2002;9:198-203.

63 Weiss SW. Alveolar soft part sarcoma: are we at the end or just the beginning of our quest? Am J Pathol 2002;160:1197-9. 